\title{
GEOMETRIC VERSUS KINETIC MODELLING APPROACH FOR CHARACTERIZING POROUS METAL FOAMS
}

\author{
ESMARI MARÉ \& SONIA WOUDBERG \\ Applied Mathematics Department, Stellenbosch University, South Africa
}

\begin{abstract}
Knowledge of the geometric and kinematic parameters of porous foams are of great importance since it is used in a wide variety of industrial multiphase flow applications that require optimal functionality, e.g. gas filters, heat exchangers and catalyst supports. The large external surface area and high porosity of metal foams provide good chemical resistance, enhanced heat and mass transfer properties and low pressure drops. Four generic geometric models will be considered to characterize the metal foam geometry, namely the cubic unit cell, tetrakaidecahedron, dodecahedron and rectangular representative unit cell (RUC) models, as well as three kinetic approaches from the literature in order to predict the specific surface area (SSA). Two sets of experimental data from the literature will then be compared to the SSA model predictions of the geometric approach and to the SSA values obtained from the kinetic approach. A comparative analysis reveals that the most geometrically complex tetrakaidecahedron model indeed provides the best correspondence with the experimental data for the SSAs, followed by the geometrically simplest RUC model. The latter model, in addition, provides accurate results for the kinetic approach. The advantage of the RUC model is that it is the only geometric model that provides both a geometric and kinetic approach, and, as a result of its relatively simple geometry it is geometrically adaptable towards anisotropy. The Klinkenberg effect will also be considered to determine the influence on the predictions of the SSAs dependency on the permeability coefficients for different fluid phases.
\end{abstract}

Keywords: metal foams, specific surface area, Klinkenberg effect, permeability, porous media, porosity, Knudsen, flow.

\section{INTRODUCTION}

Metal foams have a vast range of industrial applications. They are used in, for example, catalytic beds as compact heat sinks for power electronics, in air cooled condensers and multifunctional heat exchangers [1], in the design of aircraft wing structures in the aerospace industry [2], etc. The properties that make foams so attractive to processes requiring gas filters, heat exchangers and catalyst supports are that they exhibit a high mechanical strength, a large external surface area and a high porosity. These physical properties in turn lead to the metal foams having good chemical resistance, enhanced heat and mass transfer properties and low pressure drops [3].

Several authors in the literature have studied multiphase flow in foams, e.g. Topin et al. [4] and Edouard et al. [5]. In multiphase flow applications (in specific), metal foams have become more popular over packed beds in, for example, the petrochemical industry due to its large specific surface area and the resulting low pressure drops. This is because in industries such as the petrochemical industry, catalytic reactions between gas, liquid and solid are essential and the rates of mass transfer increases by increasing the gas and liquid contact surfaces, as well as the turbulence within the fluid phase. Furthermore, low pressure drops at high flow rates are favourable so that the productivity and selectivity of the processes can be maintained [5]. Metal foams are thus ideal for such processes. Consequently, for design and optimization purposes, the parameters of metal foams and knowledge thereof are of great importance. 
To characterize foams, morphological parameters are needed, such as the pore diameter, the strut diameter and the specific surface area (SSA). Due to the intricate nature of foams and their complex structure, it is difficult to find reliable correlations among the morphological parameters that affect transport phenomena. The micro-structure of metal foams is approximated by mainly four different geometric models, as illustrated in Fig. 1. These are the cubic unit cell model [6], the tetrakaidecahedron model [3], the dodecahedron model [7] and the rectangular representative unit cell (RUC) model [8].

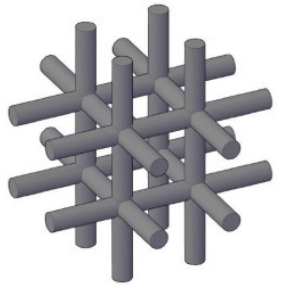

(a)

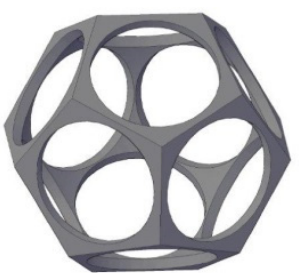

(b)

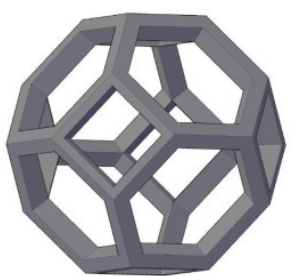

(c)

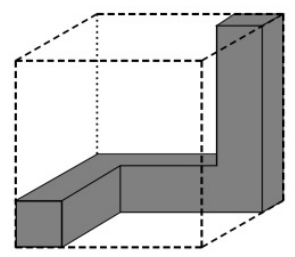

(d)

Figure 1: Geometric models for foams. (a) Cubic unit cell; (b) Dodecahedron; (c) Tetrakaidecahedron; and (d) RUC.

The objective of this paper is to investigate specifically the correlations among the morphological parameters of each of the respective model geometries by giving equations for the prediction of the SSA. Thereafter, another approach will be considered, referred to as the kinetic approach, where the transport properties of metal foams are used to determine the SSA, and are compared to the results obtained from the geometric approach. Furthermore, experimental data available in the literature for the pore-scale linear dimensions and SSA, will be compared to the model predictions of the geometric approach and to the values obtained from the kinetic approach. It will also be determined if the more complex geometries of the tetrakaidecahedron and dodecahedron models provide more accurate predictions than the simpler cubic unit cell and RUC models. Finally the influence of different fluid phases on the predicted values for the SSA will be investigated due to the Klinkenberg effect.

\section{GEOMETRIC APPROACH}

As mentioned in Section 1, the geometric models currently used in the literature to approximate the microstructure of foams are the cubic unit cell model [6], the tetrakaidecahedron model [3], the dodecahedron model [7] and the RUC model [8]. Furthermore, the specific surface area, denoted by $S_{v}$, is defined as:

$$
S_{v}=\frac{A_{s}}{V_{o}}
$$

where $A_{s}$ is the total surface area of the foam presented to the flow and $V_{o}$ is the total volume of the bed.

\subsection{Cubic unit cell model}

The cubic unit cell resembles a cube with cylindrical fibres on the edges [6]. One approach in finding expressions for the parameters of the cubic unit cell model was presented by Giani 
et al. [6]. The cell volume was computed by using the volume of a cube and the pore diameter, yielding

$$
V_{o}=d_{p}^{3}
$$

The SSA obtained in this approach in terms of the porosity, denoted by $\epsilon$, and the pore diameter is then given by

$$
S_{v}=\frac{2}{d_{p}} \sqrt{3 \pi(1-\epsilon)}
$$

Another approach in finding expressions for the parameters of the cubic unit cell model was given by Lacroix et al. [9]. In this method the volume of the cube was computed using the face diameter, defined as [10],

$$
D_{p}=d_{p}+d_{s}
$$

with $d_{s}$ denoting the strut diameter, which is the thickness in the middle of the strut and usually the thinnest part between the nodes [10]. The equation for expressing the cell volume in this approach is therefore given by

$$
V_{o}=D_{p}^{3}
$$

which leads to

$$
S_{v}=\frac{2}{d_{p}}[\sqrt{3 \pi(1-\epsilon)}-2(1-\epsilon)],
$$

for the SSA.

\subsection{Tetrakaidecahedron model}

The tetrakaidecahedron model resembles a truncated octahedron, having six square faces and eight hexagonal faces [11]. For this model, triangular strut geometry is used with an edge width $d_{s}$ and side length $l_{s}$. Similarly to the cubic unit cell model, there are two approaches to determine the SSA of the tetrakaidecahedron model. The one approach makes use of the face diameter $D_{p}[11]$ and the other the pore diameter $d_{p}[12]$.

The approach in which $D_{p}$ is used is based on the work of Gibson and Ashby [13] as used by Buciuman and Kraushaar-Czarnetzki [11]. The equation for the SSA in terms of the porosity and the pore diameter in this approach is found to be

$$
S_{v}=\frac{4.82 \sqrt{1-\epsilon}}{\left(\frac{31}{19} \sqrt{1-\epsilon}+1\right) d_{p}} .
$$

For the second approach, the SSA is determined in terms of the pore diameter $d_{p}$ as was done by Richardson et al. [12] and results in

$$
S_{v}=\frac{5.621[1-0.971 \sqrt{1-\epsilon}] \sqrt{1-\epsilon}}{d_{p}} .
$$


In the expression obtained for $S_{v}$ by Richardson et al. [12], however, the coefficient is 12.979 instead of 5.621 and it is therefore possible that there was a mistake made in the calculation of the coefficient in their equation for the SSA.

\subsection{Dodecahedron model}

The dodecahedral shape is formed by 12 pentagonal faces [7]. As observed by Bhattacharya et al. [2], the strut shape depends on the porosity of the foam, that is, the struts tend towards triangular prisms in the case of high porosity and towards cylindrical shape in the case of lower porosity. We will consider both cases of the slim dodecahedron model using the approaches followed by Huu et al. [7] to determine the SSA of the dodecahedron model at both high porosity $(\epsilon>0.9)$ with triangular strut geometry, and lower porosity $(\epsilon<0.9)$ with cylindrical strut geometry.

\subsubsection{Triangular strut geometry}

The SSA for this version of the dodecahedron model can be determined by making use of the golden ratio $(\sim 1.61803)$, denoted by $\emptyset$, and the following equation

$$
\frac{k^{2} \sqrt{15}}{\emptyset^{4}}-\frac{k^{3} \sqrt{10}}{3 \emptyset^{4}}-(1-\epsilon)=0
$$

where $k$ is defined as $d_{s} / c$ and $c$ is the length of the struts added to two times the distance between the strut and the centre mass point of the nodes [7].

The equation for the SSA in terms of the pore diameter and $k$ is then given by

$$
S_{v}=\frac{60 k}{\sqrt{5} \emptyset^{3} \sqrt{3-\emptyset} d_{p}}\left(1-\frac{1}{2} \sqrt{\frac{2}{3}} k\right)^{2},
$$

where $k$ can be solved from eqn (9).

\subsubsection{Cylindrical strut geometry}

In this version for determining the SSA, the equation for $k$, defined again as $d_{s} / c$, in terms of the porosity becomes [7]

$$
\frac{\sqrt{5} \pi}{\emptyset^{4}} k^{2}\left(1-\frac{k}{2} \sqrt{\frac{2}{3}}\right)+\frac{2 \sqrt{10}}{12 \emptyset^{4}} k^{3}-(1-\epsilon)=0 .
$$

The SSA can then be given in terms of the pore diameter and $k$, i.e.

$$
S_{v}=\frac{20 \pi k}{\sqrt{5} \emptyset^{3} \sqrt{3-\emptyset} d_{p}}\left(1-\frac{1}{2} \sqrt{\frac{2}{3}} k\right)^{2},
$$

where $k$ can be solved from eqn (11).

\subsection{RUC model}

The RUC model represents the average geometry of the complex network of interconnected struts of actual porous foams and consists of three mutually perpendicular square duct 
sections [8]. In RUC notation, $U_{f}$ denotes the fluid volume, $U_{\|}$the total streamwise fluid volume and $U_{t}$ the fluid volumes that are not bordered by solid surfaces [8]. The geometric factor $\psi$ can then be defined in terms of these parameters as [8]

$$
\psi=\frac{U_{f}}{U_{\|}+U_{t}}
$$

The geometric factor can also be obtained in terms of the porosity, yielding

$$
\frac{\psi}{\epsilon}=\frac{4}{(3-\psi)^{2}}
$$

Eqn (14) can be rewritten as an explicit function of porosity by using the Cardanic method of solving a cubic polynomial, yielding [14]

$$
\psi=2+2 \cos \left[\frac{4 \pi}{3}+\frac{1}{3} \cos ^{-1}(2 \epsilon-1)\right] .
$$

The SSA can be determined in terms of the pore diameter and the geometric factor, given by

$$
S_{v}=\frac{3}{2 d_{p}}(3-\psi)^{2}(\psi-1),
$$

where $\psi$ can be solved using eqn (15).

\section{KINETIC APPROACH}

The onset of determining the SSA of porous foams using a kinetic approach is the DarcyForchheimer equation, i.e. [15]:

$$
\frac{\Delta p}{L}=\frac{\mu}{K_{1}} q+\frac{\rho}{K_{2}} q^{2},
$$

where $\Delta p / L$ is the pressure gradient, $\mu$ is the dynamic viscosity, $\rho$ is the density of the fluid, $K_{1}$ and $K_{2}$ are the permeability coefficients of the Darcy and Forchheimer regimes, respectively, and $q$ is the superficial velocity. Eqn (17) is used to determine $K_{1}$ and $K_{2}$ from experimental data for the pressure drop. These permeability coefficients are then used to find an expression for the specific surface area. To determine $K_{1}$ and $K_{2}$, three approaches will be considered. The one approach will be based on the work of Dietrich et al. [15], another approach will be based on the work done by Huu et al. [7], and the final approach will be based on the RUC modelling approach of Woudberg and Du Plessis [8].

\subsection{Dietrich formulation}

Dietrich et al. [15] compared the Ergun equation with eqn (17), based on the assumption that the Ergun equation is also applicable to describe the pressure drop of metal foams. The Ergun equation is given by

$$
\frac{\Delta p}{L}=A \frac{\mu}{\epsilon D_{h}^{2}} q+B \frac{\rho}{\epsilon^{2} D_{h}} q^{2}
$$

where $D_{h}$ is the hydraulic diameter and $A=110$ and $B=1.45$ are empirical constants obtained for the metal foams [15]. An expression for the SSA in terms of the permeability coefficient $K_{1}$ can then be determined, given by 


$$
S_{v}=\sqrt{\frac{16 \epsilon^{3}}{110 K_{1}}}
$$

Similarly the SSA can be determined in terms of $K_{2}$, resulting in

$$
S_{v}=\frac{4 \epsilon^{3}}{1.45 K_{2}} \text {. }
$$

\subsection{Huu formulation}

The approach followed by Huu et al. [7] is similar to that of Dietrich et al. [15], but instead of using the Ergun equation defined in terms of $D_{h}$ they defined it in terms of $d_{p}$, that is

$$
\frac{\Delta p}{L}=E_{1} \frac{\mu(1-\epsilon)^{2}}{\epsilon^{3} d_{p}^{2}} q+E_{2} \frac{\rho(1-\epsilon)}{\epsilon^{3} d_{p}} q^{2},
$$

where $E_{1}=150$ and $E_{2}=1.75$. To avoid using a geometric approach as Huu et al. did in defining the pore diameter, it was decided to rather let $d_{p}=D_{h}$, as was done in the Dietrich approach. The SSA was then determined in terms of the permeability coefficients, yielding

$$
S_{v}=\sqrt{\frac{16 \epsilon^{5}}{150 K_{1}(1-\epsilon)^{2}}}
$$

which is the SSA in terms of $K_{1}$, and

$$
S_{v}=\frac{4 \epsilon^{4}}{1.75 K_{2}(1-\epsilon)}
$$

in terms of $K_{2}$.

\subsection{RUC formulation}

In the RUC approach, expressions for $K_{1}$ and $K_{2}$ are determined as shown in the study of Woudberg and Du Plessis [8]. An Ergun-type equation for porous foams is determined from the RUC model and simplified to give the following expression for the pressure gradient:

$$
\frac{\Delta p}{L}=\frac{9 \psi^{2}(\psi-1)(3-\psi)^{2}}{\epsilon^{2} d_{p}^{2}} \mu q+\frac{(\psi-1)(3-\psi)^{2} \psi^{2} c_{d}}{8 \epsilon^{3} d_{p}} \rho q^{2},
$$

where $\psi$ is as defined in eqn (15) and $c_{d}$ is the interstitial form drag coefficient with a value of $c_{d}=2$. Defining $d_{p}$ to be equal to $D_{h}$, as in the previous approaches, once again leads to an equation for the SSA in terms of $K_{1}$, i.e.

$$
S_{v}=\frac{4 \epsilon}{3 \psi(3-\psi) \sqrt{K_{1}(\psi-1)}}
$$

and $K_{2}$, i.e.

$$
S_{v}=\frac{16 \epsilon^{4}}{K_{2}(\psi-1)(3-\psi)^{2} \psi^{2}} .
$$




\section{GEOMETRIC AND KINETIC APPROACHES COMPARED TO EXPERIMENTAL DATA}

The influence of the porosity on the SSA equations obtained in the geometric approach in dimensionless form, that is $S_{v} d_{p}$, is shown in Fig. 2. The dimensionless SSA predictions obtained using the geometric approach is compared to the experimental data of Dietrich et al. [15] and Incera Garrido et al. [10]. It can be seen that all of the equations predict that the SSA decreases as the porosity increases, which makes physical sense. The SSA predicted by the dodecahedron model were split into two porosity ranges, 0.7 to 0.9 and 0.9 to 1 for the cylindrical and triangular strut geometry, respectively. Although Fig. 2 shows that the most accurate predictions obtained when compared to this experimental data are those from the tetrakaidecahedron model, which is considered to be the most complicated of the geometric models, the second most accurate model is the prediction determined from the RUC model, which is considered to be the simplest geometric model of the four considered. The dodecahedron model is the third most accurate (although the results are very similar to that of the RUC) and the cubic unit cell the fourth. It should be emphasized that these findings are purely based on the experimental data considered in this study. Comparison with other data may produce different results.

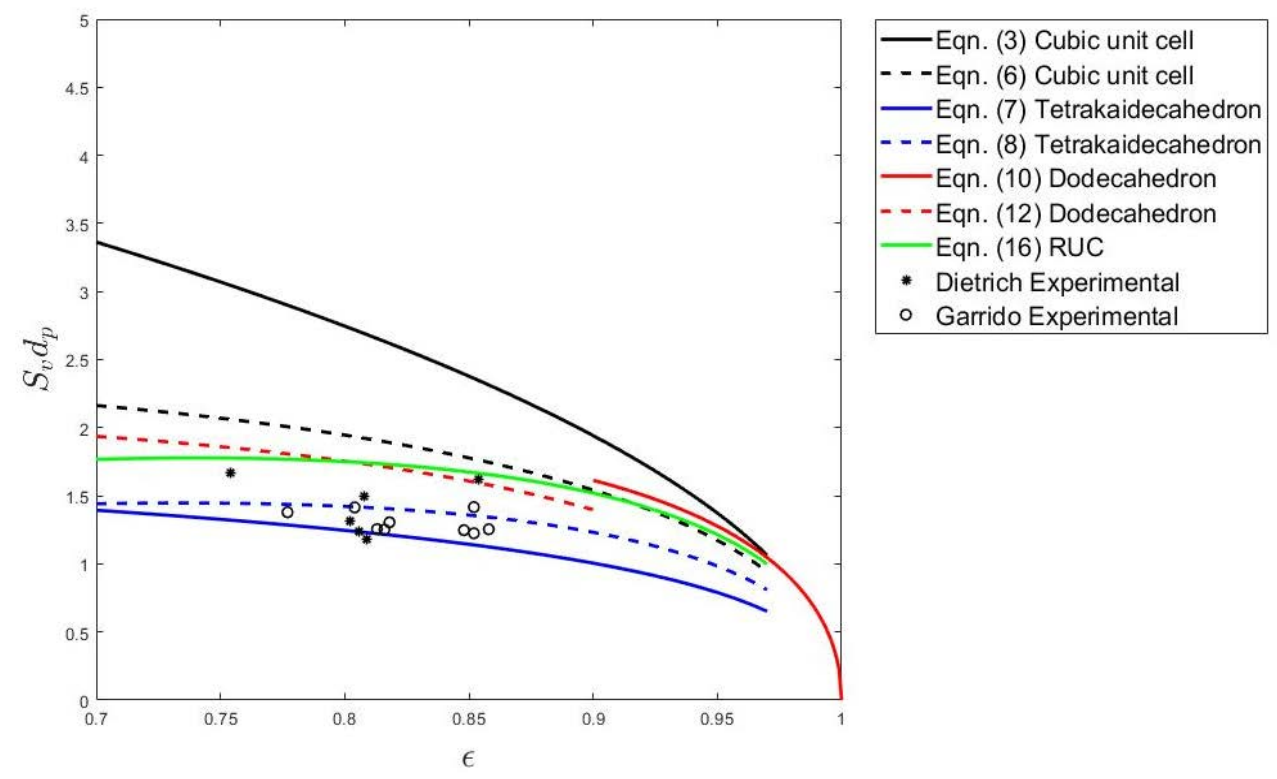

Figure 2: Dimensionless SSA versus porosity predicted by all the Geometric approach models compared to experimental data from the literature.

To evaluate the equations for the SSA obtained from the kinetic approaches, two sets of experimental data for $K_{1}$ and $K_{2}$ were considered. One set was that of Richardson et al. [12] and the other that of Liu et al. [16], shown in Fig. 3. The experimental data of Dietrich and Garrido correspond well with the values of the dimensionless SSA obtained from the RUC and Dietrich approaches. The values obtained from the Huu approach does, however, not appear to be a good approximation when compared to the experimental data. 


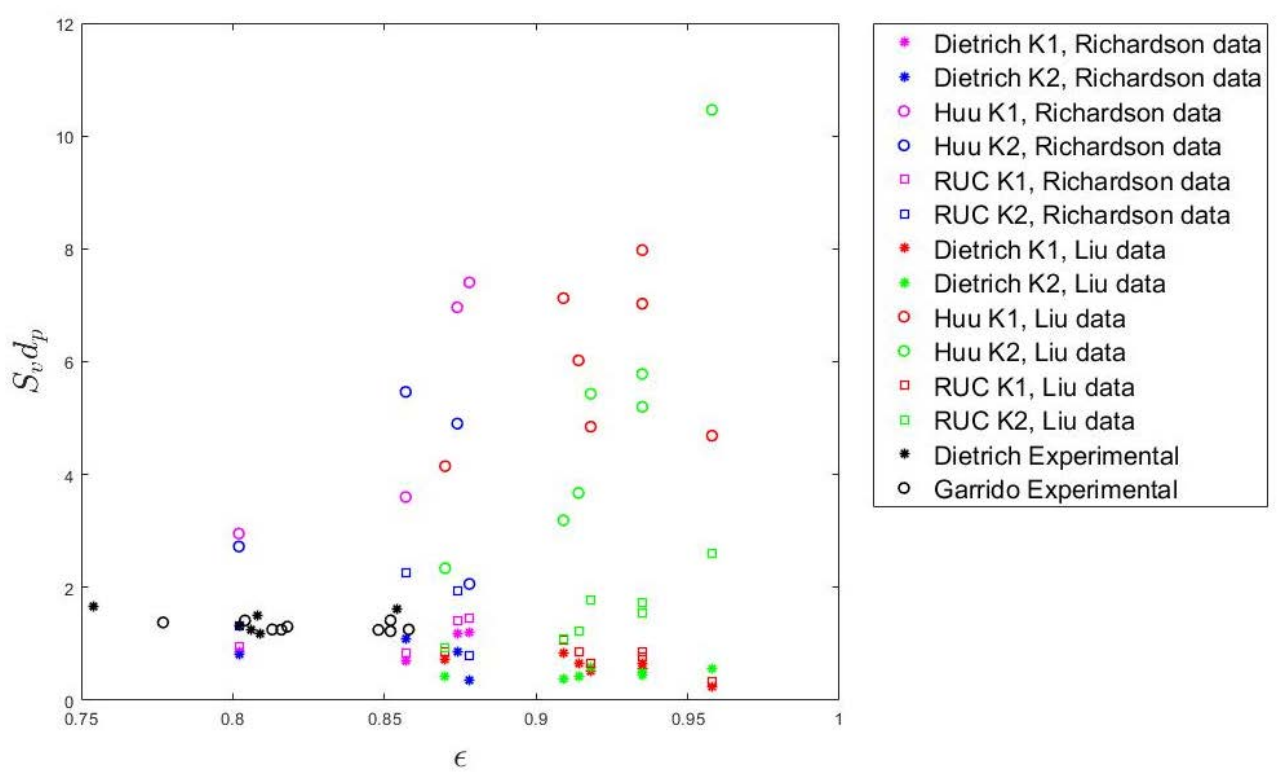

Figure 3: Dimensionless SSA values versus porosity obtained from all the Kinetic approaches compared to experimental data from the literature.

To address the question of whether the more complex geometries of the tetrakaidecahedron and dodecahedron models provide more accurate predictions than the simpler cubic unit cell and RUC models, the comparisons of the geometric models with the experimental data are considered. If the tetrakaidecahedron and dodecahedron models are considered together as the more complex models, the complex models provide more accurate predictions than the simpler RUC and cubic unit cell models. If the four models are considered individually where the complexity of the models in decreasing order are the tetrakaidecahedron, dodecahedron, RUC and the cubic unit cell model, the more complex models do not provide more accurate predictions due to the RUC model providing slightly more accurate predictions than the dodecahedron model.

\section{FLUID PHASE DEPENDENCE DUE TO THE KLINKENBERG EFFECT}

Gas and liquid flow in porous media may produce different permeability values. Reasons for these differences include the fact that gas is much more compressible than liquid, adsorption occurring in porous media of low porosity and the Klinkenberg effect [17]. In this study only the Klinkenberg effect will be considered and how it influences the flow of gas through porous media when compared to liquid flow.

The Klinkenberg effect is related to the slip condition of the flow at the walls of the pore. The Klinkenberg effect becomes significant at low or near atmospheric pressure, when the permeabilities of the flow are low and when the molecular mean free path, denoted by $\lambda$, is comparable to the dimensions of the pore [17].

Due to this effect, the flow deviates from Darcy's law, which in turn leads to an adjustment of the Navier-Stokes equation to implement a slip-boundary condition. To determine when the boundary condition should be implemented, a ratio is used, known as the Knudsen number and, given by [17] 


$$
K_{n}=\frac{\lambda}{D_{h}} .
$$

When $K_{n} \leq 10^{-3}$ the continuum assumption is valid in the Navier-Stokes equation and a no slip-boundary condition can still be implemented. When $10^{-3}<K_{n}<10^{-1}$ the continuum approach is still valid, but a slip-boundary condition at the pore walls needs to be incorporated in the Navier-Stokes equation and when $K_{n} \geq 10^{-1}$ the Navier-Stokes equation cannot be used since the gas would then exhibit non-continuum effects [17]. [17]

An expression relating the liquid and gas permeabilities involving the Knudsen number is

$$
K_{g}=K_{l}\left[1+12 \frac{(2-\sigma)}{\sigma} K_{n}\right],
$$

where $\sigma$ is the tangential momentum accommodation coefficient (the fraction of molecules which undergo diffuse reflection at the pore walls [17]).

In the Kinetic approach in Section 4, the experimental results obtained from the Richardson et al. and Lui et al. data were from measuring gas flow through foams. The permeability coefficients in the Kinetic approach was that of liquid and need to be expressed in terms of the permeability coefficient of gas to determine whether the Klinkenberg effect affects the SSA results, or alternatively, whether different fluid phases would lead to different SSA results when determined as in the Kinetic approach. Rearranging eqn (28) therefore leads to an expression of $K_{l}$ in terms of $K_{g}$, i.e.

$$
K_{l}=\frac{K_{g}}{1+12 \frac{(2-\sigma)}{\sigma} K_{n}} .
$$

The tangential momentum accommodation coefficient $\sigma$ ranges mostly between 0.2 and 1 and tends towards 0 for a smooth surface and towards 1 for a rough surface, since it depends on the properties of the wall. The SSA versus porosity values for the case of gas flow plotted in Fig. 4 are those obtained from substituting eqn (29) into the Dietrich and RUC formulations, i.e. eqns (19) and (25), respectively. Due to the results of the Huu formulation not performing well when compared to the Dietrich and RUC formulations, the Huu formulation was not added in this investigation of fluid phase effects on the SSA results. For each formulation $\sigma$ equalling $0.2,0.5$ and 1.0 were plotted to determine the effect of the surface smoothness on the results. The Knudsen number was taken to be equal to $10^{-3}$ to determine the significance of the Klinkenberg effect on the SSA values in the case of the lower bound of estimated slip-flow occurrence.

It can be observed in Fig. 4 that the predictions for the dimensionless SSA decreases with increasing $\sigma$-values. All the Dietrich formulation values, obtained using the Richardson data are pink, blue and green, in decreasing order, corresponding to $\sigma$-values of $0.2,0.5$ and 1 , respectively, for each porosity value in the data set. Physically this also makes sense, since it would be expected that the slip-flow at the boundaries would decrease for surfaces that are less smooth and as a result the Klinkenberg effect would decrease. The closest prediction to the experimental values is provided by the cases where $\sigma=0.2$. This can be observed more clearly when the predictions for the tetrakaidecahedron geometric model, which was estimated to represent the data most accurately among the geometric models, is compared to the SSA estimations. 


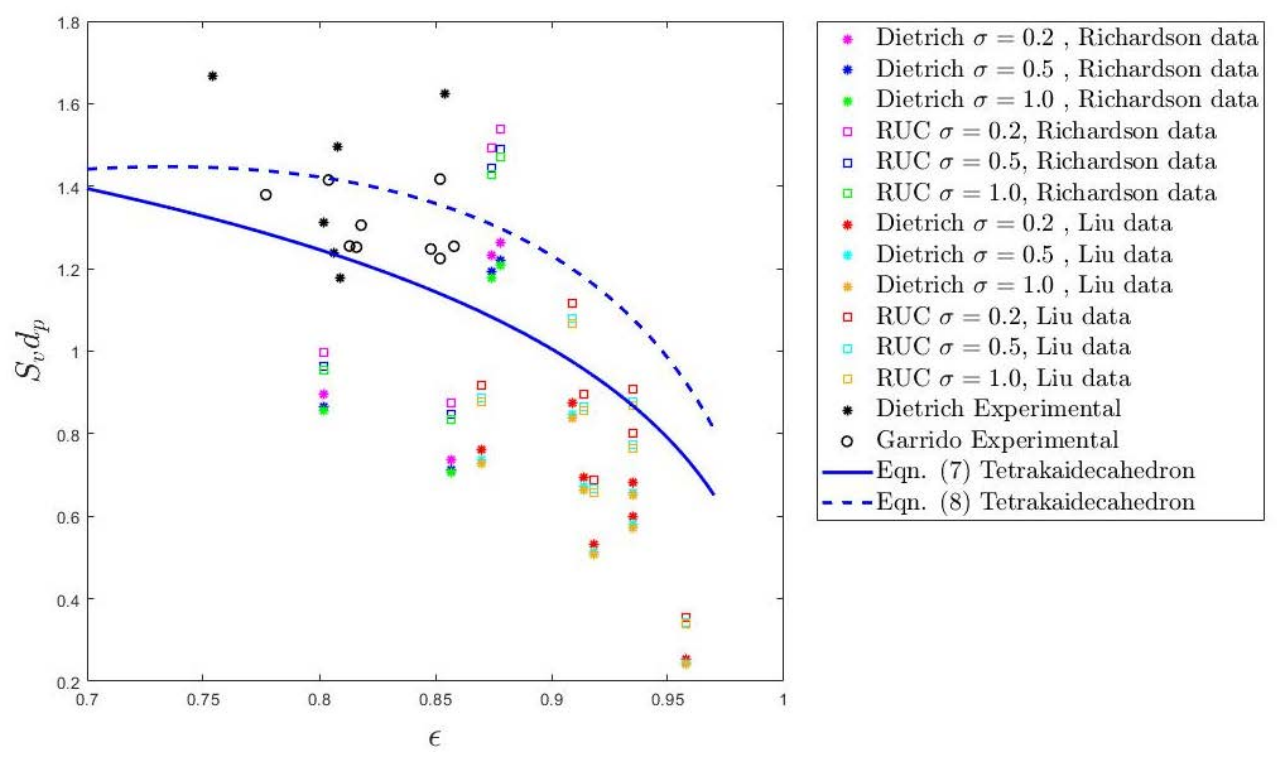

Figure 4: Dimensionless SSA values versus porosity obtained from the Kinetic approaches and implementation of the Klinkenberg effect compared to experimental data from the literature and the tetrakaidecahedron model predictions for $K_{n}=10^{-3}$.

The predicted values for the SSA shown in Fig. 4 has a relative percentage difference range of $0.6 \%$ to $5.3 \%$ for all three $\sigma$-values when compared to the predicted values obtained in the Kinetic approach. It furthermore shows a better correspondence with the trend of the experimental data when $K_{n}$ is incorporated. When the upper bound of estimated slip-flow occurrence along with a continuum approach is considered, i.e. when $K_{n}=10^{-1}$, the percentage difference range is $48.3 \%$ to $243.5 \%$, which is significantly higher, as expected.

In conclusion, the Klinkenberg effect can have an influence if the Knudsen number is high enough. The question would now, however, be whether the Knudsen number can reach the required interval for it to have an effect. In Miguel and Serrenho [18] the influence of gas properties on the permeability coefficient in porous media was investigated. They determined that air has a mean free path of $\lambda=70 \mathrm{~nm}$ and for helium $\lambda=200 \mathrm{~nm}$. Miguel and Serrenho [18] then calculated $K_{n}$ for sand and fibrous material, by using the latter $\lambda$-values and eqn (27). Once again letting $D_{h}$ be equivalent to $d_{p}$, we can determine the approximate order of Knudsen number in the data used in this study. For the data of Richardson et al. [12] and Lui et al. [16] the order of magnitude of $d_{p}$ is $10^{-3}$. The Knudsen number for air flow would therefore be of an order of approximately $10^{-5}$, which is too small to truly have an effect. Similarly, the Knudsen number for helium flow through the metal foams would be of an order of approximately $10^{-4}$, which is also insignificant. Other data obtained for stainless steel fibres by Manzo et al. [19] has an order of magnitude of $10^{-6}$ for $d_{p}$, which results in the Knudsen number for air and helium flow being of the order of magnitude $10^{-2}$ and $10^{-1}$, respectively. Since foams are considered to fall under the category of fibres and not granular media [20], it can therefore be concluded that the Klinkenberg effect can affect the SSA results for foams of small enough diameter, i.e. for pore diameters of the order of $10^{-6}$ or less. 


\section{CONCLUSIONS}

The geometric model that compared most favourably to the experimental data for the specific surface area in the geometric approach was the geometrically most complex tetrakaidecahedron model. In the kinetic approach the RUC and Dietrich predictions were the most accurate. The Klinkenberg effect did have a measure of influence in the data considered in this study, but it was regarded as negligible due to the relatively large pore sizes. For other finer materials, i.e. for pore diameters of the order of $10^{-6}$ or less., however, the effect is expected to produce more significant deviations from liquid flow.

\section{ACKNOWLEDGEMENTS}

The support of the South African DST-NRF Centre of Excellence in Mathematical and Statistical Sciences (CoE-MaSS) towards this research is hereby acknowledged. Opinions expressed and conclusions arrived at, are those of the authors and are not necessarily to be attributed to the CoE.

\section{REFERENCES}

[1] Mancin, S., Zilio, C., Cavallini, A. \& Rossetto, L., Pressure drop during air flow in aluminum foams. International Journal of Heat and Mass Transfer, 53(15-16), pp. 3121-3130, 2010.

[2] Bhattacharya, A., Calmidi, V. \& Mahajan, R., Thermophysical properties of high porosity metal foams. International Journal of Heat and Mass Transfer, 45(5), pp. 1017-1031, 2002.

[3] Inayat, A., Freund, H., Zeiser, T. \& Schwieger, W., Determining the specific surface area of ceramic foams: The tetrakaidecahedra model revisited. Chemical Engineering Science, 66(6), pp. 1179-1188, 2011.

[4] Topin, F., Bonnet, J., Madani, B. \& Tadrist, L., Experimental analysis of multiphase flow in metallic foam: Flow laws, heat transfer and convective boiling. Advanced Engineering Materials, 8(9), pp. 890-899, 2006.

[5] Edouard, D., Lacroix, M., Pham, C., Mbodji, M. \& Pham-Huu, C., Experimental measurements and multiphase flow models in solid $\mathrm{SiC}$ foam beds. American Institute of Chemical Engineers, 54(11), pp. 2823-2832, 2008.

[6] Giani, L., Groppi, G. \& Tronconi, E., Mass-transfer characterization of metallic foams as supports for structured catalysts. Industrial and Engineering Chemistry Research, 44(14), pp. 4993-5002, 2005.

[7] Huu, T., Lacroix, M., Pham Huu, C., Schweich, D. \& Edouard, D., Towards a more realistic modeling of solid foam: Use of the pentagonal dodecahedron geometry. Chemical Engineering Science, 64(24), pp. 5131-5142, 2009.

[8] Woudberg, S. \& Du Plessis, J., An analytical Ergun-type equation for porous foams. Chemical Engineering Science, 148, pp. 44-54, 2016.

[9] Lacroix, M., Nguyen, P., Schweich, D., Pham Huu, C., Savin-Poncet, S. \& Edouard, D. Pressure drop measurements and modeling on SiC foams. Chemical Engineering Science, 62(12), pp. 3259-3267, 2007.

[10] Incera Garrido, G., Patcas, F., Lang, S. \& Kraushaar-Czarnetzki, B., Mass transfer and pressure drop in ceramic foams: A description for different pore sizes and porosities. Chemical Engineering Science, 63(21), pp. 5202-5217, 2008.

[11] Buciuman, F. \& Kraushaar-Czarnetzki, B., Ceramic foam monoliths as catalyst carriers. 1. Adjustment and description of the morphology. Industrial and Engineering Chemistry Research, 42(9), pp. 1863-1869, 2003. 
[12] Richardson, J., Peng, Y. \& Remue, D., Properties of ceramic foam catalyst supports: pressure drop. Applied Catalysis A: General, 204(1), pp. 19-32, 2000.

[13] Gibson, L.L. \& Ashby, M.F. (eds), Cellular Solids, Structure and Properties, Pergamon Press: Oxford, 1988.

[14] Wilms, J.M., On the hydrodynamic permeability of foamlike media. MSc Eng. thesis, Stellenbosch University, South Africa, 2006.

[15] Dietrich, B., Schabel, W., Kind, M. \& Martin, H., Pressure drop measurements of ceramic sponges-determining the hydraulic diameter. Chemical Engineering Science, 64(16), pp. 3633-3640, 2009.

[16] Liu, J., Wu, W., Chiu, W. \& Hsieh, W., Measurement and correlation of friction characteristic of flow through foam matrixes. Experimental Thermal and Fluid Science, 30(4), pp. 329-336, 2006.

[17] Woudberg, S. \& Du Plessis, J.P., Predicting the permeability of very low porosity sandstones. Transport in Porous Media, 73, pp. 39-55, 2008.

[18] Miguel, A.F. \& Serrenho, A., On the experimental evaluation of permeability in porous media using a gas flow method. Journal of Physics D: Applied Physics, 40, pp. 68246828, 2007.

[19] Manzo, G., Wu, Y., Chase, G. \& Goux, A., Comparison of nonwoven glass and stainless steel microfiber media in aerosol coalescence filtration. Separation and Purification Technology, 162, pp. 14-19, 2016.

[20] Jackson, G.W. \& James, D.F., The permeability of fibrous porous media. The Canadian Journal of Chemical Engineering, 64, pp. 364-374, 1986. 